\title{
Pre-Nucleation induced Fast Ordering in Block-Copolymer Films with Dynamic Zone Annealing
}

Maninderjeet Singh ${ }^{1}$, Joseph Strzalka², Sushil Satija ${ }^{3}$, Alamgir Karim $^{1, *}$

${ }^{1}$ Department of Chemical and Biomolecular Engineering, University of Houston, Houston TX 77204 akarim3@central.uh.edu

${ }^{2}$ X-Ray Science Division, Argonne National Laboratory, Argonne, IL 60439 strzalka@anl.gov

${ }^{3}$ NIST Center for Neutron Research, NIST, Gaithersburg, MD 20878 satija@nist.gov

The long ordering times scales along with number of complex processing steps may hinder the realization of use of block copolymer self-assembly in next generation lithography and other applications. Here, we demonstrate a simple and fast route for vertical assembly of lamellae forming PS$b$-PMMA Block Copolymer (L-BCP) on unmodified substrates, by utilizing the effect of pre-nucleation in as cast films, imparted by suitable casting solvent followed by dynamic thermal gradients based annealing technique known as Cold Zone Annealing (CZA). We demonstrate the effect of different casting solvents and film casting methods to understand the fundamentals of generation of prenucleated morphology during glass formation in film casting as characterized initially by AFM followed by GISAXS. For PS-b-PMMA films with a high fraction of pre-nucleated in-plane microphase-separation, vertical orientation of $L-B C P$ is achieved at CZA speeds ranging from $0.05 \mathrm{~mm} / \mathrm{s}$ to $0.5 \mathrm{~mm} / \mathrm{s}$ which translates to annealing times ranging from 2 minutes to 10 seconds in BCP films with thickness range from $1 \mathrm{~L}_{0}$ (domain length-37 nm in this case) to more than $20 \mathrm{~L}_{0}$. The effect of pre-nucleation in as cast films and the final morphology are quantified using AFM and GISAXS, including using in-situ GISAXS with a CZA apparatus placed on-line at the Advanced Photon Source's 8-ID-E beam line at Argonne National Laboratory, IL wherein the X-ray beam traces the sample across the thermal gradient profile point-bypoint. 